\title{
Estilos de aprendizaje y metodologías de enseñanza en estudiantes de obstetricia
}

\author{
E. Villalobos-Alarcón a, M. Guerrero-Faquíez ${ }^{a}$, R. Pérez-Villegas a , A. Avendaño-Veloso a, \\ A. Ceballos-Morales a, J. Ortiz-Contreras ${ }^{a}$, C. Parra-Ponce ${ }^{\text {b }}$
}

Introducción. Si consideramos que todos los estudiantes no son iguales y aprenden de manera diferente, es necesario identificar sus estilos de aprendizaje (EA) y las metodologías que faciliten el proceso enseñanza-aprendizaje. Este estudio pretende diagnosticar los EA predominante en estudiantes de obstetricia y correlacionarlos con las metodologías educativas aplicadas. Sujetos y métodos. Se realizó un estudio observacional, correlacional y transversal, durante el período 2006-2007, en 72 estudiantes de obstetricia de la Facultad de Medicina, Universidad de Concepción, Chile. Las variables estudiadas y correlacionadas fueron estilo de aprendizaje y metodologías de enseñanza. Se utilizó el Cuestionario de EA Honey-Alonso (CHAEA), y se realizó una encuesta de satisfacción de las metodologías que facilitaron el aprendizaje. Para el análisis estadístico se utilizó la prueba chi al cuadrado, y la correlación entre ambas variables se obtuvo con el coeficiente de rho de Spearman. Resultados. Los estilos predominantes fueron el reflexivo y teórico $(p<0,0001)$. Las metodologías de enseñanzas que más facilitaron el aprendizaje fueron el uso de modelos y la clase expositiva. No se encontró correlación estadística entre los EA y las metodologías de enseñanza utilizadas. Conclusión. La determinación de los EA en los estudiantes de la salud es fundamental para seleccionar las metodologías de enseñanza más adecuadas.

Palabras clave. Cuestionario CHAEA. Estilo de aprendizaje. Metodologías de enseñanza.

\section{Learning styles and teaching methodologies in obstetric students}

Introduction. Considering that all students are not equal and learn differently, is necessary to identify their learning styles and methodologies that facilitate the teaching- learning process. This study aims to diagnose learning styles prevalent in Obstetric students and correlation with educational methodologies applied. Subjects and methods. Observational and cross-correlation study, during the period 2006-2007 in 72 Obstetric students, Faculty of Medicine, University of Concepción, Chile. The variables were learning style and teaching methodologies. The instrument used was the questionnaire learning styles Honey-Alonso (CHAEA) and satisfaction survey methodologies that helped the learning. The statistical differences were conducted using chi-square test and the correlation between two variables was obtained with the coefficient of Spearman's rho. Results. The dominant were reflexive and theoretical styles ( $p<0,0001$ ). Methodologies for more facilitated learning were the use of models and the expositive class. No correlation was found between statistical learning styles and teaching methodologies used. Conclusion. The identification of learning styles in student health is essential to select the most appropriate teaching methodologies.

Key words. CHAEA questionnaire. Learning style. Methodology teaching.

\section{Introducción}

El concepto 'aprender a aprender', definido como el conocimiento y destreza necesarios para aprender con efectividad en cualquier situación en que uno se encuentre, permitió el desarrollo del planteamiento de las teorías de estilos de aprendizaje (EA) [1].

Los EA son los rasgos cognitivos, afectivos y fisiológicos que sirven de indicadores relativamente estables de cómo los estudiantes perciben, interaccionan y responden en sus ambientes de aprendizaje [2].

\footnotetext{
Departamento de Obstetricia y Puericultura. Facultad de Medicina.

b Departamento de Psicología. Facultad de Ciencias Sociales. Universidad de Concepción. Ciudad Concepción, Chile.
}

Correspondencia Dra. Eva Villalobos Alarcón. Departamento de Obstetricia y Puericultura. Facultad de Medicina. Universidad de Concepción. Barrio Universitario, s/n. Casilla 160. 4030000 Ciudad Concepción, Chile.

Fax

56-41-2204837.

E-mail

evillal@udec.cl

Investigación financiada por el Proyecto de Mejoramiento de la Calidad de la Docencia de Pregrado n. 06-074. Dirección de Docencia, Universidad de Concepción, Chile. 
Respecto a la categorización de los estilos de aprendizaje, existe una diversidad de clasificaciones. La más utilizada es la de Honey y Mumford y, posteriormente, Alonso, quienes propusieron cuatro categorías fundamentales: estilos activo, reflexivo, teórico y pragmático, las cuales se aplicarán en este estudio $[1,3]$. Estos EA, aunque relativamente estables, pueden ser modificados, y es responsabilidad de los docentes ayudar a los estudiantes a descubrir su estilo y aprender a adaptarlo a las experiencias de cada situación $[2,4]$.

El conocimiento de la forma cómo se expresan los estilos de aprendizaje en los grupos de estudiantes de los cursos universitarios podría ser una herramienta docente muy útil para generar e identificar metodologías apropiadas, adaptando el estilo de enseñanza del profesor con el fin de conseguir un mejor rendimiento académico [5], a la vez que permite diseñar métodos de evaluación más apropiados para comprobar el progreso de los estudiantes [6].

La investigación sobre los estilos cognitivos ha tenido grandes implicaciones para la docencia, al brindar evidencias que sugieren que el acomodar los métodos de enseñanza a los estilos preferidos de los estudiantes, puede traer consigo una satisfacción mayor de éstos y también una mejora en los resultados académicos [7].

En el área de la salud se cuenta con métodos de enseñanza distintos que se aplican a los estudiantes durante las prácticas clínicas como: clases expositivas, uso de modelos, análisis de casos clínicos y empleo de tecnologías de la información y la comunicación (TIC), los cuales desconocemos si responden a las necesidades de los estudiantes según los estilos de aprendizaje. Por tanto, el objetivo de este estudio es diagnosticar los estilos de aprendizaje predominante en los estudiantes de obstetricia y correlacionarlos con las metodologías educativas aplicadas en el proceso enseñanza-aprendizaje.

\section{Sujetos y métodos}

Estudio observacional, correlacional y transversal, durante el período 2006-2007, en 72 estudiantes de obstetricia de la Facultad de Medicina, Universidad de Concepción, Chile.

El instrumento utilizado para medir los EA fue el Cuestionario de estilos de aprendizaje Honey-
Tabla I. Distribución de estudiantes según metodologías de aprendizaje $(n=72)$.

\begin{tabular}{lc}
\hline Clase expositiva & $22(30,6 \%)$ \\
\hline Uso de modelos & $33(45,8 \%)$ \\
\hline Análisis de casos clínicos & $14(19,4 \%)$ \\
\hline $\begin{array}{l}\text { Uso de tecnologías de la } \\
\text { información y la comunicación }\end{array}$ & $3(4,2 \%)$ \\
\hline
\end{tabular}

Alonso (CHAEA) [1]. Dicho cuestionario se realizó por un encuestador de forma simultánea, con la participación voluntaria de los estudiantes.

Se realizó una encuesta validada de satisfacción del usuario (escala Likert) para conocer la metodología que les facilitaba más su aprendizaje. Esta encuesta fue aplicada al final del período.

Para procesar la información, se elaboró una base de datos en programa Excel y se realizó un análisis estadístico con el programa SPSS versión 13.0, y se aplicó la prueba de chi alcuadrado para obtener las diferencias estadísticas de las variables, considerándose significativo valores $p<0,05$.

$\mathrm{El}$ análisis de correlación entre los EA y las metodologías de enseñanza se realizó a través del análisis no paramétrico, de cálculo del coeficiente de correlación rho de Spearman.

\section{Resultados}

Se analizó los EA de los 72 estudiantes, 67 mujeres $(93,1 \%)$ y 5 hombres $(6,9 \%)$. El promedio de edad fue de 20,47 $\pm 0,93$ años.

El perfil predominante de los estudiantes fue el reflexivo $(58,3 \% ; n=42) \mathrm{y}$, en segundo lugar, el teórico $(18,1 \% ; n=13)$. Sólo ocho alumnos poseen EA pragmático $(11,1 \%)$ y seis activo $(8,3 \%)$. La diferencia entre los EA fue estadísticamente significativa $(p<0,0001)$. Hubo un estudiante en cada una de las combinaciones siguientes: activo-pragmático, reflexivo-teórico y teórico-pragmático.

La tabla I muestra la distribución de estudiantes según la preferencia de metodologías de enseñanza; así, las metodologías con mayor aceptación 
Tabla II. Distribución de los estudiantes según estilos de aprendizaje y metodologías utilizadas.

\begin{tabular}{lcccccccccc}
\hline Estilo de aprendizaje & \multicolumn{2}{c}{$\begin{array}{c}\text { Uso de } \\
\text { modelos }\end{array}$} & $\begin{array}{c}\text { Clase } \\
\text { expositiva }\end{array}$ & $\begin{array}{c}\text { Análisis de } \\
\text { casos clínicos }\end{array}$ & $\begin{array}{c}\text { Utilización } \\
\text { de TIC }\end{array}$ & $\begin{array}{c}\text { N. o de } \\
\text { estudiantes }\end{array}$ \\
\cline { 2 - 10 } & $n$ & $\%$ & $n$ & $\%$ & $n$ & $\%$ & $n$ & $\%$ & $n$ & $\%$ \\
\hline Activo & 3 & 50 & 1 & 16,7 & 2 & 33,3 & 0 & 0 & 6 & 100 \\
\hline Reflexivo & 21 & 50 & 13 & 31 & 6 & 14,2 & 2 & 4,8 & 42 & 100 \\
\hline Teórico & 4 & 31 & 6 & 46 & 3 & 23,1 & 0 & 0 & 13 & 100 \\
\hline Pragmático & 4 & 50 & 2 & 25 & 1 & 12,5 & 1 & 12,5 & 8 & 100 \\
\hline Activo-pragmático & 1 & 100 & 0 & 0 & 0 & 0 & 0 & 0 & 1 & 100 \\
\hline Reflexivo-pragmático & 0 & 0 & 0 & 0 & 1 & 100 & 0 & 0 & 1 & 100 \\
\hline Teórico-pragmático & 0 & 0 & 0 & 0 & 1 & 100 & 0 & 0 & 1 & 100 \\
\hline Total & 33 & 45,8 & 22 & 30,6 & 14 & 19,4 & 3 & 4,2 & 72 & 100 \\
\hline
\end{tabular}

TIC: tecnologías de la información y la comunicación.

por los estudiantes fueron el uso de modelos y la clase expositiva ( 45,8 y $30,6 \%$, respectivamente). La diferencia estadística entre estas metodologías y el análisis de casos clínicos y la utilización de TIC fue significativa $(p<0,0001)$.

Con respecto a la correlación entre los EA predominantes y las metodologías utilizadas, entre los estudiantes con estilo reflexivo, el 50\% prefirió el uso de modelos como método de aprendizaje y el $31 \%$ prefirió la clase expositiva.

Entre los alumnos con estilo teórico, el 31\% prefirió el uso de modelos como método de aprendizaje, el $46 \%$ prefirió las clases expositivas y el 23,1\% los análisis de casos clínicos (Tabla II).

El coeficiente de correlación rho de Spearman, calculado para estos datos, fue $p=-0,142$, lo que indica que no hay una correlación entre los EA y las metodologías utilizadas $(p=0,235)$.

\section{Discusión}

Según los resultados obtenidos en nuestro estudio, los EA predominantes fueron el reflexivo y el teórico, similar a lo obtenido por Highifield [8] y Canalejas [4], y diferente a Correa et al [3].
Parece ser que los estudiantes del área de la salud, al inicio de su formación, priorizan la reflexión y la teoría antes de realizar actividades clínicas hospitalarias, las cuales exigirían estilos más prácticos o activos.

En un futuro es posible estudiar si el acercamiento a actividades netamente clínicas produce alguna modificación en la tendencia a otros estilos, si se considera que los EA son sólo relativamente estables o si pueden sufrir modificaciones a lo largo del tiempo [9].

La falta de correlación entre los EA y las metodologías preferidas por los alumnos podría explicarse según lo planteado por Bermesolo [10], quien indica la necesidad de multiplicar las estrategias de enseñanza en relación con los diversos EA de los estudiantes.

\section{Bibliografía}

1. Alonso C, Gallego D, Money P. Estilos de aprendizaje. 4 ed. Bilbao: Mensajero; 2000.

2. Alonso C. Estilos de aprendizaje y estudiantes universitarios. In Gallego D, Alonso C, eds. Estilos de aprender 
y estilos de enseñar [curso de doctorado]. UNED; 20012002.

3. Correa J. Identificación de los estilos de aprendizaje en los estudiantes de fisiología del ejercicio de la Facultad de Rehabilitación y Desarrollo Humano. Rev Cienc Salud 2006; 4 (Supl1): S41-53.

4. Canaleja M, Martínez M, Pineda M, Vera M, Sotop M, Martín A. Estilos de aprendizaje en los estudiantes de enfermería. Educ Med 2005; 8: 33-40.

5. Ordóñez F, Rosety-Rodríguez M, Rosety-Plaza M. Análisis de los estilos de aprendizaje predominante en los estudiantes de ciencia de la salud. Enfermería Global, 2003. URL: http://www.um.es/eglobal/3/pdf/03c04.pdf [14.01.2008].
6. Lochart, D, Schmeck RR. Learning styles and classroom evaluation methods: different strokers for different folks. Coll Stud J 1983; 117: 94-100.

7. Nunnan D. Language teaching methodology: a textbook for teachers. New York: Prentice Hall; 1991.

8. Highfield ME. Learning styles. Nurse Educator 1988; 30-3.

9. Ceballos B, Barahona A. El diagnóstico de los estilos de aprendizaje: un instrumento valioso para apoyar la formación en los estudiantes de enfermería. Enfermería Global, 2003. URL: http://www.um.es/eglobal/3/pdf/03c04. pdf [14.01.2008].

10. Bermesolo J. Cómo aprenden los seres humanos. Mecanismos psicológicos del aprendizaje. Santiago de Chile: Ediciones Universidad Católica de Chile; 2005. 